\title{
ECONOMIE SOLIDAIRE ET DEMOCRATIE PARTICIPATIVE LOCALE
}

\section{Abdourahmane NDIAYE}

L'insertion, portée par les acteurs de l'économie solidaire, associe accompagnement social et activité économique en faveur des publics en difficulté. Par cette approche, les structures d'insertion par l'activité économique (SIAE) ne considèrent pas le social comme un résidu de l'économique (Ndiaye, 2008). Bien au contraire, le paradigme porté par les courants théoriques de l'économie solidaire est de considérer l'activité économique comme un moyen permettant de réaliser un projet politique et non comme une fin qui déboucherait sur une accumulation de richesses. L'idée de promouvoir des processus économiques collectifs contextualisés, complexes et flexibles puise son essence dans l'accompagnement et l'anticipation des mutations sociales (Pecqueur, 2000). Ces processus économiques collectifs «situés » ont comme cadre spatial le territoire que l'on considère comme un objet problématique pluridimensionnel. Ses différentes dimensions convergent de manière plus ou moins conflictuelle pour engendrer de nouvelles modalités de création de ressources, apporter des solutions sociopolitiques et socioéconomiques inédites (Di Méo, 1998) et répondre à ce que Rosanvallon (1995) appelle la nouvelle «question sociale ${ }^{1}$. Mais arrivera-t-on à un «ré-encastrement» du marché, de l'économie dans la société ?

En ouvrant un espace propice à l'agir ensemble au sens de Crozier, Friedberg (1977), les acteurs de l'économie solidaire réaffirment leur volonté transformationnelle. On peut néanmoins s'interroger sur la capacité de ceux-ci à forger un nouveau contrat social.

\footnotetext{
${ }^{1}$ Selon Rosanvallon (1995), l'avènement d'une nouvelle question sociale coïncide avec la fin des «Trente Glorieuses ». Au début des années quatre-vingt, la croissance du chômage et l'apparition de nouvelles formes de pauvreté ont permis de constater que la crise de l'État-providence avait changé de nature en rendant inadaptées les anciennes méthodes de gestion du social.
} 
L'action collective, initiée par le truchement du plan local pour l'insertion et l'emploi (PLIE), dans les espaces interstitiels des territoires, permet-elle l'avènement d'une démocratie participative locale ? Le fait que les usagers, associations, entreprises et pouvoirs publics se retrouvent au sein de ces espaces pour proposer, négocier, délibérer et valider les orientations et modalités de l'insertion est-il suffisant pour parler d'un nouveau contrat social? L'objectif de ce travail est de présenter les résultats partiels d'une enquête réalisée par nos soins dans le territoire du PLIE des Graves, ce qui permet de répondre à ce questionnement.

\section{CADRE METHODOLOGIQUE DE LA DEMOCRATIE PARTICIPATIVE LOCALE AU SEIN DES TERRITOIRES}

1.1. Origine et filiation de la démocratie participative locale en France

La démocratie participative locale est, selon le Centre d'éco-développement et d'initiative sociale (Cédis) et l'Association pour la démocratie et l'éducation locale et sociale (Adels) (2007, pages 10-11) reprenant la définition d'Antoine Bevort, "non seulement un idéal politique moderne, mais aussi un modèle performant. Plus les citoyens sont en capacité de s'exprimer et de délibérer sur le devenir de la cité, plus une communauté est en capacité d'agir pour ces fins. Plus un régime politique prend en compte les attentes de ses membres, plus il est efficace. La participation n'est ni l'autre de la représentation, ni son simple supplément d'âme, mais la force exigeante qui anime de son imagination créatrice la démocratie ». Plus qu'un idéal, la démocratie participative locale est la clé d'une exigence nouvelle pour laquelle les capacités individuelles articulées aux capacités collectives développent et entretiennent le capital social. La démocratie participative locale est officialisée en France durant les années 1990 avec l'institutionnalisation du développement durable. C'est une orientation politique, influencée par le rapport Brundtland et l'Agenda 21, qui vise à "élargir notre 
représentation de la proximité en l'intégrant dans une vision globale associant économie soutenable, environnement préservé et solidarité sociale, dans une échelle de temps longs» (Braillon, 2007). Le développement durable, au travers de l'Agenda 21, a influencé d'une certaine manière l'ancrage d'une démocratie participative locale dont l'émergence est le résultat d'une triple filiation en France.

Tout d'abord, la première filiation de ce triptyque est le développement local qui marque une dynamique endogène, autocentrée et ascendante de reprise en main des destinées de la vie locale par les habitants. Les tenants du développement local ont l'impression d'avoir été abandonnés par les politiques publiques qui se détournent des régions dévitalisées pour ne plus s'intéresser qu'à celles qui gagnent (Benko, Lipietz, 1992). Souvent organisés contre ou sans les autorités publiques locales (Raibaud, à paraître), les acteurs du développement local veulent apporter une réponse pragmatique devant les difficultés croissantes introduites par l'après-fordisme, le postmodernisme face à la faiblesse de la réaction des pouvoirs publics. L'émergence en France d'un développement local peut être analysée comme le résultat de tentatives des « derniers survivants qui ne veulent pas mourir sur place, ceux qui n'attendent plus rien d'en haut, qui ont tourné le dos à l'État...» qui créent des structures locales, principalement constituées d'associations de développement local. Ces schémas de développement local s'inspirent des slogans à la mode dans les pays en développement durant les années soixante-dix, du genre : « on ne développe pas, on se développe !».

L'autogestion municipale est la deuxième filiation de la démocratie participative locale. Issue d'une critique de la gauche "anarchiste », elle prend appui sur le constat d'une «instrumentalisation» du citoyen dans la vie démocratique, qui n'est sollicité qu'au moment des élections. Partant des limites du fonctionnement de la démocratie représentative, des groupes locaux issus du monde associatif montent une argumentation revendiquant une intervention permanente du citoyen dans la vie locale. Celle-ci passe par un accès à une information objective et pluraliste; une 
institutionnalisation des consultations et des instances pour la co-élaboration, la co-construction et la cogestion des projets locaux ; l'exigence de comptes rendus de la part des mandataires à leurs mandants (l'accountability).

La troisième filiation est liée aux principes qui soustendent la politique de la ville. Le délabrement des « grands ensembles » observé durant les années 1970 dans les territoires urbains appelait à leur réhabilitation. Celle-ci était considérée comme une opportunité pour les habitants des «quartiers sensibles» de s'approprier les processus initiés. Les habitants auto-organisés initient des projets de quartier en partenariat avec des animateurs, des travailleurs sociaux, des responsables associatifs. Ceux-ci créent une opportunité dialectique entre un mouvement ascendant (bottom-up) qui prend sa source des autoorganisations des citoyens en destination des pouvoirs publics et des institutions et un mouvement descendant (top-down) qui part des pouvoirs publics et leurs institutions vers les citoyens.

1.2. Cadre théorique et méthodologie de la «participation»

D'une manière plus générale, le processus participatif local est appréhendé comme le moteur des stratégies de développement local si tant est qu'il donne la possibilité de la combinaison d'une forte appropriation locale et d'une approche large qui inclut la prise en compte des institutions et la «bonne gouvernance». L'approche «participative» se veut être autant un levier de démocratie locale et de proximité dans sa dimension politique qu'une stratégie de développement local dans une perspective socioéconomique. Pour appréhender la participation, nous examinerons les trois approches suivantes.

\subsubsection{L'approche de la Banque mondiale}

En économie du développement, le cadre de référence du concept de «participation» adopté est issu des travaux d'Edgerthon et al. (2000, page 2) qui définissent la participation comme le processus par lequel les parties prenantes influencent les initiatives de développement, 
les décisions et les ressources qui les affectent et en partagent le contrôle ${ }^{2}$. Le processus participatif passe par quatre phases. Dans la première phase, la communication de l'information où les flux d'informations des décideurs, à sens unique, sont à destination du public. La deuxième phase structure la consultation qui occasionne un flux d'informations allant dans les deux sens. Lors de la troisième phase, la collaboration permet l'organisation d'un échange de vues, de stratégies. La quatrième et dernière phase autorise la concertation sur les politiques et la prise en main de leur destin par les citoyens qui nécessitent la mise en place d'un environnement favorable à l'agir ensemble. Si pour les inspirateurs de l'approche de la Banque mondiale, il existe quatre niveaux de compréhension et d'exercice de la participation, pour d'autres, notamment Arnstein (1969), de l'école de Chicago, il y aurait huit degrés d'appropriation de la participation ${ }^{3}$. La mobilisation du processus participatif est considérée comme la clé de voûte de la réussite des politiques de lutte contre la pauvreté. Ce nouveau cadre s'appuie sur le Document de stratégie de réduction de la pauvreté et tente de faire de la «participation» des acteurs un enjeu de tout premier plan (Ndiaye, 2009). L'émergence de nouveaux acteurs tels que les élus locaux issus des lois sur la décentralisation et les leaders d'opinions mandatés par les mouvements sociaux locaux, nationaux et internationaux intervient à un moment où on redécouvre les vertus de l'État développeur. D'un cadre conceptuel et méthodologique dans lequel l'État est le problème et le marché la solution, on a évolué vers un cadre plus nuancé dans lequel on accepte les échecs et les défaillances des marchés et où l'État doit jouer un rôle structurant en

\footnotetext{
${ }^{2}$ Sur un essai d'analyse systématique du concept de «participation » tel qu'il est utilisé dans les nouvelles stratégies internationales de lutte contre la pauvreté et l'endettement par la Banque mondiale, voir Cling (2003).

${ }^{3}$ (1) « Manipulation totale de la population par les pouvoirs publics »; (2) «système assistanciel distributif, appliqué à des populations maintenues volontairement dans un état de passivité »; (3) « information»; (4) « consultation»; $\quad$ (5) « conciliation»; (6) «association »; (7) « délégation de pouvoir»; (8) « contrôle par les citoyens ».
} 
partenariat avec les acteurs sociaux (Dercon, 2004). Il est conçu comme un cadre général dont l'objectif est de redéfinir l'outillage analytique de la Banque mondiale considéré comme inadapté pour répondre aux problèmes des sous-développements en y introduisant une dimension politique. Cette vision paradigmatique de la Banque mondiale s'appuie sur les notions de consultation, de consensus, de transparence, d'accountability et de bonne gouvernance. Tout en étant des partenaires au développement, les acteurs locaux représentent des protagonistes (instrumentalisés) de l'État qui reste seul maître à bord. De fait, le paradigme de la Banque mondiale ne s'appuie pas suffisamment sur la capacité transformationnelle des acteurs locaux.

1.2.2. L'approche par les capabilités ou l'agencéité (agency)

La participation rejoint d'une certaine manière le concept d'agencéité ou d'agentivité développé dans Dubois, Brouillet, Bakhshi et Duray-Soundron (2008). L'approche de l'agencéité, tout en donnant une certaine résonance aux travaux de Sen (2000) et de Nassbaum (2000) concernant les capabilités individuelles et collectives, insiste sur la capacité d'organisation intentionnelle, d'action et d'anticipation individuelle et collective, et surtout de transformation sociale. La participation, partant de l'agencéité individuelle et collective, se décline à travers quatre formes. Ces déclinaisons constituent autant de stades d'approfondissement de l'appropriation, de l'augmentation ou la potentialisation (empowerment) et de la responsabilisation démocratique. Il est donc question d'un processus qui mobilise les acteurs du développement local, notamment les élus locaux, les représentants de la société civile et les publics, y compris les usagers eux-mêmes. Cette démarche participative, qui doit s'appuyer sur les structures politiques existantes en vue de les renforcer en tant que lieu de concertation et de délibération sur les problèmes de développement social, s'inscrit dans une logique d'ancrage de la démocratie et de construction d'une nouvelle citoyenneté. Le processus participatif suppose donc que les acteurs de l'économie 
solidaire «organisés» et capables d'anticipation adressent une demande aux pouvoirs publics locaux perçus comme un agent incontournable du développement et devant maîtriser un agenda permettant de réaliser le projet de société entériné par les élections. Tout en étant sectorielle, l'approche participative se veut transversale et doit porter les germes d'une nouvelle citoyenneté dans laquelle les changements proviendraient des interactions entre citoyens (usagers ou bénéficiaires), acteurs sociaux du développement local et élus locaux. Cette approche, tout en insistant sur les dimensions positives de l'empowerment et de la responsabilisation démocratique semble minorer la dimension spatiale du développement local.

\subsubsection{Le paradigme du site symbolique}

Les territoires peuvent être conçus comme des sites au sens de la théorie des sites telle qu'elle a été développée par Zaoual (Daghri, Zaoual, 2008 ; Zaoual, 1999). Le paradigme des sites symboliques ou «sitologie» prend «la précaution de postuler que le site est une entité immatérielle, multidimensionnelle et dynamique ». En mobilisant l'interactionnisme symbolique et la relativité, la théorie du site cherche à intégrer fortement la dimension territoriale non pas d'un point de vue physique mais plutôt en référence aux acteurs et au sens implicite de leurs pratiques. Le paradigme des sites réhabilite le «sens commun» et permet de relier les différentes composantes du site : la «boite à outils » qui implémente les comportements et les modèles d'actions, la «boite conceptuelle» qui structure les connaissances et la «boite noire» qui en constitue l'ordre des croyances (Machrafi, 2008). La théorie du site symbolique entretient des affinités avec l'approche du «milieu innovateur» développée par Aydalot (1986) qui fait l'hypothèse que la dynamique socioéconomique ne préexiste pas aux milieux locaux, mais qu'elles sont sécrétées par eux. Ces approches mettent en avant les différentes formes d'interdépendances qui se tissent dans le territoire et interviennent dans les dynamiques socioéconomiques qui incorporent des éléments et des acteurs très variés. Néanmoins, la notion de territoire est 
malaisée à manier, pour les géographes mais davantage pour les socioéconomistes. Il constitue un objet problématique pluridimensionnel. La définition de son périmètre est loin d'être évidente. S'il semble établi que le territoire est le résultat d'un découpage administratif, celui-ci peut se rapprocher ou à l'inverse prendre ses distances par rapport à des évocations historiques collectives, une géographie ou une revendication d'appartenance identitaire. La définition des contours physiques des territoires est non seulement laborieusement obtenue, mais elle souffre de fluctuations, selon les temporalités et les légitimités convoquées. Le passage d'une commune à une communauté de communes ou de la commune au canton en sont des illustrations éloquentes.

Les trois approches participatives mobilisées semblent à notre sens complémentaires et peuvent déboucher sur une tentative de justification socioéconomique de la participation. Les acteurs sont considérés comme ayant une rationalité limitée et que leurs interactions ne sont pas uniquement guidées par un calcul économique. En référence à «la liberté de bien-être [qui] renvoie à la liberté dont dispose une personne de réaliser une vie de qualité à travers la satisfaction de son propre bien-être. La liberté d'agent fait référence à la liberté qu'a une personne d'adhérer à des valeurs et de concevoir des buts généraux, et non pas seulement des objectifs liés à son propre bien-être. » (Panet, Duray-Soundron, 2008, page 18). Cette liberté de bien-être combinée au site symbolique donnerait une "agencéité située », sorte de synthèse dialectique entre l' "agencéité » et la «sitologie» qui autorise l'empowerment individuel et collectif. Appliquée à la participation au sein d'un territoire, cela débouche sur une certaine défection et une prise de parole au sens de Hirschman (1995) ${ }^{4}$. Comment cela se traduit-il concrètement au sein du territoire du PLIE des Graves?

\footnotetext{
${ }^{4}$ Dans une communication originale, Chochoy (à paraître) présente les fondements possibles de l'économie solidaire à partir du triptyque exit-voice-loyalty de Hirschman. Pour une présentation plus large de la défection et de la prise de parole, voir Hirschman (1995).
} 


\section{PLIE ET DEMOCRATIE PARTICIPATIVE}

2.1. Contexte et cadre méthodologique de la recherche

\subsubsection{Le contexte}

Le PLIE des Graves couvre la commune de Bègles et la Communauté de Communes de Montesquieu (CCM), qui sont situées sur la rive gauche du département de la Gironde en région Aquitaine. L'élément fédérateur, le ciment de cette communauté de destin est la proximité «idéologique ». En effet, entourée de communes de droite (Bordeaux, Talence, Villenave d'Ornon), le maire de Bègles a préféré s'associer avec les treize communes de gauche de la CCM: Ayguemorte les Graves, Beautiran, Cabanac et Villagrains, Cadaujac, Castres Gironde, Isle-Saint-Georges, La Brède, Léognan, Martillac, Saint-Médard-d'Eyrans, Saint-Morillon, Saint Selve et Saucats ${ }^{5}$. Cette alliance entre communes de gauche conduite par le premier maire écologiste d'une ville de plus de 5000 habitants pourrait laisser penser qu'elle est de nature à davantage favoriser l'éclosion d'une démocratie participative locale. Nous nous gardons de faire une telle hypothèse, nous pensons plutôt que c'est l'espace d'agir ensemble forgé par le PLIE qui est de nature à encourager l'émergence et le renforcement d'une démocratie participative locale. Nous pensons que les clivages gauche/droite qui traversent la vie politique française ne semblent pas, en la matière, être des facteurs déterminants. La sensibilité politique n'a donc rien à voir avec l'attachement à des valeurs démocratiques.

Ce territoire est peuplé de 54000 habitants et bénéficie de l'implantation d'une zone d'activités de 4000 entreprises qui salarient près de 15000 personnes, dont plus de $70 \%$ dans le secteur des services et du commerce. Paradoxalement une bonne partie du territoire est consacrée aux vignobles.

\footnotetext{
${ }^{5}$ Dans une perspective longue, ces logiques d'alliances pourraient être fragilisées par le fait que les communes, les communautés de communes et même les cantons, sont des entités politiques dans lesquelles l'alternance politique s'expérimente. Ces expérimentations donnent cours à des renversements de majorités et l'irruption de questions et d'alliances nouvelles.
} 
En 2006, environ 1800 personnes (dont 600 jeunes peu ou pas qualifiés, 860 allocataires du RMI, 680 chômeurs de longue durée) résidant sur le territoire du PLIE des Graves ont des difficultés à accéder à un emploi et relèvent potentiellement du dispositif du PLIE ${ }^{6} .952$ demandeurs d'emplois sont élus au bénéfice des services du PLIE et peuvent être caractérisés par les éléments suivants : $59 \%$ de femmes (contre $51 \%$ en moyenne nationale); avec deux tiers d'adultes et un tiers de jeunes; $43 \%$ de très faible niveau de qualification et $20 \%$ de niveau bac et plus; près de $40 \%$ de cette population est bénéficiaire du RMI ; $45 \%$ des bénéficiaires du PLIE résident dans des quartiers relevant du Contrat Urbain de Cohésion Sociale (CUCS) ${ }^{7}$.

Comme nous avons fait l'hypothèse forte que les résultats obtenus dépendent de la qualité des collaborations et des partenariats entre les acteurs, nous allons réinterroger les instances délibératives, les dynamiques, les stratégies et les jeux des acteurs.

\subsubsection{Cadre et modalités de recueil de l'information}

L'essentiel des structures d'insertion du territoire du PLIE des Graves se trouve sur la commune de Bègles qui est le moteur de la dynamique du PLIE. L'enquête sur la démocratie participative locale est une rubrique d'une enquête sur la capacité du PLIE des Graves à insérer durablement les personnes ayant des difficultés à accéder à un emploi. Elle a été effectuée à Bègles, sur un échantillon composé d'une association de développement local (ADELE), d'une association chantier d'insertion (Arcins Environnement Services), d'une entreprise d'insertion (Arcins Entreprise), d'une régie de quartier (La Gabare) et d'un institut de formation (INFOSUP). Le choix de cet échantillon est guidé par l'implication des acteurs dans l'insertion par l'activité économique sur le territoire du PLIE des Graves. Outre ces acteurs institués, il nous a semblé légitime d'interroger les acteurs non

\footnotetext{
${ }^{6}$ Certaines personnes appartenant à deux catégories (par exemple RMI et chômeurs de longue durée), les chiffres ne peuvent s'additionner sans précautions.

${ }^{7}$ Voir Ndiaye (à paraître).
} 
institués que sont les bénéficiaires des dispositifs d'insertion. L'intérêt porté aux acteurs non institués permet de mettre à nu certaines limites du fonctionnement des institutions. Ces limites révélées enrichissent les délibérations et les connaissances sur le PLIE. En effet, le dispositif PLIE est érigé pour répondre aux problématiques des chômeurs les plus éloignés d'un emploi, les chômeurs de longue ou de très longue durée. Or, ces acteurs non institués se sentent (et sont de fait) souvent marginalisés et le PLIE constitue selon eux le genre de dispositif sensé réparer les injustices dont ils sont victimes. Aussi à ce niveau, ils ressentent leur exclusion des discussions et des délibérations comme une des pires injustices. Ainsi, nous avons rencontré une quinzaine de chômeurs de longue et de très longue durée. Leur prise en compte en surnombre dans l'échantillon était une manière de tenir compte de cet état de fait et d'en corriger symboliquement les effets pervers.

Le guide d'entretien qui sous-tend cette enquête, qui a été menée entre février et avril 2008, poursuit les deux objectifs principaux suivants. Le premier objectif principal du guide d'entretien est de montrer comment les acteurs de l'économie solidaire contribuent à faire émerger une démocratie participative locale au travers de la lutte contre le chômage. Le guide se focalise d'abord sur les définitions de la démocratie participative retenues par les responsables de structures et leurs bénéficiaires. Ensuite, on cherche à recenser les conditions nécessaires et suffisantes pour son existence, à identifier les instances de régulation et de délibération et à évaluer leur efficacité. Enfin, il s'agit d'identifier les facteurs de blocage et les conditions optimales d'une démocratie participative. Le deuxième objectif principal est d'identifier les objectifs et d'évaluer les résultats du PLIE des Graves et donc ceux des acteurs de l'économie solidaire opérant sur le territoire. Cet objectif permet de voir les articulations mais aussi les conflits et décalages entre ce qui est officiellement annoncé et les réalités du terrain. Paradoxalement, certains des acteurs ne se sentent pas du tout concernés par les résultats affichés par le PLIE même s'ils y contribuent. Ils analysent cet affichage par le PLIE de leurs propres résultats comme une sorte d'usurpation et le moyen par lequel il capte l'essentiel 
des subventions publiques. Le temps nécessaire pour l'administration du guide d'entretien est variable. Les entretiens ont duré entre une heure et une heure et demie.

\subsection{Instances délibératives et dynamique des acteurs}

Les directions des SIAE et les chômeurs interrogés dans le cadre de cette enquête ont donné des définitions de la démocratie participative qu'on peut synthétiser dans le tableau 1. Les représentations de la démocratie participative locale semblent être influencées par le positionnement et la légitimité des acteurs rencontrés. Les dirigeants associatifs proches de la majorité municipale pensent que la démocratie participative est un exercice permettant aux acteurs de pouvoir partager les diagnostics et la prise de décision.

Tableau 1: Conceptualisation de la démocratie participative locale par les acteurs associatifs

\begin{tabular}{|c|l|}
\hline \multirow{4}{*}{$\begin{array}{c}\text { Qu'est ce que la } \\
\text { démocratie } \\
\text { participative? }\end{array}$} & $\begin{array}{l}\text { 1. Une participation à la prise de } \\
\text { décision, un engagement fort du } \\
\text { mouvement associatif qui soit } \\
\text { respecté par les élus locaux }\end{array}$ \\
\cline { 2 - 2 } & $\begin{array}{l}\text { 2. Donner la possibilité aux acteurs } \\
\text { associatifs de s'exprimer, de se faire } \\
\text { entendre, de participer à l'élaboration } \\
\text { des politiques. Que cette possibilité } \\
\text { ne soit pas réservée à une élite }\end{array}$ \\
\cline { 2 - 2 } & $\begin{array}{l}\text { 3. Etre tourné vers les habitants, } \\
\text { lutter contre les exclusions }\end{array}$ \\
\cline { 2 - 2 } & $\begin{array}{l}\text { 4. Identifier et faire émerger un } \\
\text { besoin collectivement et y apporter } \\
\text { une solution par un diagnostic } \\
\text { partagé. Procéder à l'élaboration } \\
\text { commune d'un cahier des charges }\end{array}$ \\
\hline
\end{tabular}

Source : Ndiaye, 2008.

De par leurs représentations, les militants associatifs proches de la majorité municipale considèrent la participation comme une dynamique relevant exclusivement de la responsabilité et de la volonté des élus. Ce qui semble s'expliquer par le fait que leur légitimité y prend sa source. En revanche, les autres acteurs pensent plutôt que la démocratie participative doit 
être l'occasion de s'exprimer, de se faire entendre par les élus locaux, de les interpeller et de délibérer. Ces derniers considèrent que la démocratie locale doit être portée par la société civile et que le simple fait qu'il existe des instances participatives n'exclut pas la possibilité de penser celles-ci comme des appendices ou de simples guichets pilotés par les élus. Comment qualifier le fonctionnement d'instances composées de membres soigneusement sélectionnés par la municipalité et qui délibèrent à huis clos sur les affaires du territoire ? En réinterrogeant les délibérations, les délibérants et leurs bases de légitimité, nous posons la question fondamentale de la gouvernance. Plus que le fruit des délibérations, il s'agit de s'intéresser et d'examiner les modalités de celles-ci. En effet, «La coexistence en une même instance d'élus, de conseillers spécialement désignés, d'associatifs et de simples habitants ne garantit nullement la capacité de chacun de ces groupes à s'exprimer sur un même pied d'égalité ou à parler le même langage » Blondiaux (2001 : 46). L'ouverture des espaces d'agir ensemble ne signifie pas la disparition d'une communication verticale entre gouvernants et gouvernés, puisque les acteurs associatifs considèrent que la démocratie participative est la possibilité pour les habitants, quelle que soit leur posture, de se faire entendre et de contribuer à concevoir, à produire et à mettre en œuvre les politiques publiques. En formulant ces observations, certains acteurs associatifs semblent confirmer l'idée que ce sont les populations les plus fragiles, les plus exposées aux conséquences d'éventuelles décisions du PLIE, qui ont les probabilités les plus faibles d'y être représentées. Il s'agit des acteurs non institués dont le point de vue peut pourtant enrichir considérablement les débats et les solutions envisagées. Les bénéficiaires du PLIE (chômeurs et allocataires du RMI) quant à eux pensent que la démocratie participative locale est une parodie, dès lors qu'elle ignore la majorité à travers la quelle, elle puise sa légitimité. Comment, selon eux, concevoir une démocratie qui exclut le peuple ? Ce questionnement nous conduit à recenser et analyser les facteurs de blocage de la démocratie participative locale. 
Tableau 2: Facteurs de blocage de la démocratie participative locale

\begin{tabular}{|c|l|}
\hline \multirow{4}{*}{$\begin{array}{c}\text { Quels sont les facteurs de } \\
\text { blocage de la démocratie } \\
\text { participative? }\end{array}$} & $\begin{array}{l}\text { 1.la consultation des bénéficiaires } \\
\text { est instrumentale, leur avis ne } \\
\text { change rien }\end{array}$ \\
\cline { 2 - 2 } & $\begin{array}{l}\text { 2. les convictions et les croyances } \\
\text { ne sont pas partagées par toutes les } \\
\text { parties prenantes }\end{array}$ \\
\cline { 2 - 2 } $\begin{array}{l}\text { 3.le PLIE bloque les énergies, il } \\
\text { instaure une hiérarchie et en } \\
\text { constitue le sommet, les logiques } \\
\text { institutionnelles souhaitables sont } \\
\text { donc en conflit avec les logiques } \\
\text { clientélistes établies }\end{array}$ \\
\hline
\end{tabular}

Source : Ndiaye, 2008.

La tentative de conceptualisation de la démocratie participative locale à partir des SIAE montre que selon les trajectoires empruntées, la dynamique participative peut être perçue à la fois comme une revendication émergeant de la base citoyenne et une cooptation (reconnaissance par le haut) des acteurs associatifs par les élus locaux. Le Conseil d'administration du PLIE, présidé par le maire de la commune de Bègles est l'instance suprême de délibération. Son existence ne préfigure pas forcément la vigueur d'une démocratie participative locale. Si en effet le PLIE regroupe un certain nombre d'acteurs associatifs et d'élus, il n'en demeure pas moins vrai qu'il a été initié par la volonté des élus locaux.

Le PLIE des Graves est structuré par le Conseil d'administration d'ADELE, présidé par le Maire de Bègles, secondé par le Président de la Communauté de Communes de Montesquieu. Ces deux élus constituent la caution politique du portage du PLIE et de sa place privilégiée dans la mise en œuvre des politiques locales de l'emploi. Cette structuration montre la dimension hautement politique de cette instance délibérative. Le Conseil d'administration est épaulé dans sa fonction de prise de décision par le Comité de pilotage. Les directeurs de structures d'insertion font leur apparition dans le dispositif au niveau opérationnel. Les acteurs de l'économie solidaire, quant à eux, sont confinés aux fonctions d'exécution des actions. 
L'appréciation du fonctionnement de la démocratie participative par les acteurs de l'insertion par l'activité économique présents au PLIE est soit sans objet, considérant qu'il est exemplaire, soit très critique. Ceux qui portent la critique du mode de fonctionnement de la démocratie participative arguent de la forte probabilité d'une «caporalisation » des instances délibératives par les élus locaux. Néanmoins, si quasiment tous les acteurs pensent que le PLIE fonctionne démocratiquement, certains considèrent que l'absence de participation des usagers ou des bénéficiaires en constitue une faiblesse.

Les acteurs associatifs craignent une «technocratisation» du dispositif ou à l'inverse son « instrumentalisation» par les élus locaux. Ce risque est d'autant plus présent que certains acteurs pensent que le PLIE tétanise les énergies en créant artificiellement une hiérarchie dont il s'accaparerait le leadership.

\subsection{Performances du modèle}

Les performances du modèle peuvent être évaluées selon deux critères. Le premier critère fait référence aux résultats du PLIE en terme de lutte contre le chômage de longue durée. Il permet de présenter des données quantitatives et de les analyser. Le deuxième critère cherche à intégrer fortement la dimension territoriale en faisant référence aux jeux des acteurs et à leur sens implicite. On verra si le «sens commun» du site est réhabilité.

\subsubsection{Les résultats quantitatifs du PLIE des Graves}

Les sorties du dispositif PLIE sont considérées comme «positives» lorsqu'elles débouchent sur un emploi durable ou une formation. Un emploi est dit durable s'il concerne un contrat à durée indéterminée ou un contrat à durée déterminée long de plus de 18 mois. Dans le territoire du PLIE des Graves, $47 \%$ de ceux qui $\mathrm{y}$ sont accueillis en ressortent négativement. Sur ces sorties "négatives", 25 à $30 \%$ démissionnent ou abandonnent en n'étant pas ou peu mobilisés et/ou omettent d'honorer les rendez vous pris avec leurs référents d'insertion. Le reste des sorties «négatives» 
s'explique par des déménagements et des problèmes de santé ou de maternité.

En revanche, le taux de sorties «positives » évalué à $53 \%$ dépasse largement la moyenne nationale, estimée à $34 \%$. Au 30 juin 2006, $60 \%$ des bénéficiaires de parcours avaient un contrat de travail avec $38 \%$ de $\mathrm{CDI}^{8}$ ou de CDD longs, $29 \%$ en $\mathrm{CDD}^{9}$ courts et missions d'intérim et $33 \%$ en contrats aidés (contrat d'avenir, contrat d'accompagnement dans l'emploi) ${ }^{10}$. Ces résultats pourraient s'expliquer par plusieurs raisons. Tout d'abord, il faut constater que le PLIE, en suivant un petit nombre de personnes, opère un traitement de qualité. Ensuite, le recrutement des bénéficiaires du PLIE se fait en fonction de critères «internes » qui ne sont pas toujours clairement communiqués. En s'intéressant en priorité à ceux qui sont le plus proche d'un emploi, le PLIE maximise sa capacité à avoir des résultats satisfaisants. L'élargissement des publics cibles à des jeunes diplômés ayant des difficultés d'accès à un premier emploi stable ${ }^{11}$, à des femmes à l'issue de leur congé parental constitue un élément d'explication des résultats positifs obtenus. Enfin, la réussite du PLIE est fonction de la disponibilité et de l'écoute des élus locaux, de la qualité partenariale du tissu économique (entreprises, associations, administrations publiques et parapubliques) qui s'est concrétisée par la création d'un club d'entrepreneurs, de l'efficacité des actions mises en œuvre mais aussi du savoir faire et de la connaissance de terrain de l'équipe d'animation et de gestion. En vertu de la clause d'insertion, la loi exige que $5 \%$ des marchés publics soient attribués aux structures d'insertion par l'activité économique (SIAE), alors que les pouvoirs publics locaux attribuent $7 \%$ aux structures d'insertion du PLIE des Graves. Ces résultats sont d'autant plus appréciables qu'ils sont obtenus avec un minimum de ressources financières. Les tensions financières vont aller s'amplifiant puisqu'il est prévu une baisse des

\footnotetext{
${ }^{8} \mathrm{CDI}$ : Contrat à durée indéterminée.

${ }^{9} \mathrm{CDD}$ : Contrat à durée déterminée.

${ }^{10}$ Un CDD est dit court lorsqu'il n'excède pas 6 mois.

${ }^{11}$ Un emploi est considéré comme stable s'il porte sur un contrat à durée indéterminée ou un contrat à durée déterminée pouvant aller audelà de 18 mois.
} 
subventions du fonds social européen de près de $35 \%$ entre le premier conventionnement (2001-2006) et le deuxième conventionnement (2008-2012), passant de 650000 à 460000 euros pour 952 personnes à insérer. Ce qui signifie que les pouvoirs publics décentralisés doivent jouer un rôle prépondérant dans le financement, puisqu'en dernier ressort, ils sont garants de la cohésion sociale sur leur territoire. Dans le même temps, les acteurs des SIAE considèrent les contrats aidés comme appréciables pour les parcours d'insertion mais déplorent le désengagement de l'État qui va de pair avec la multiplication des acteurs participant aux financements et le manque d'harmonisation de leurs procédures. L'encadrement de plus en plus resserré et la lourdeur des procédures conjugués à une orientation des dispositifs et des instruments vers les logiques de fonctionnement du système libéral affaiblissent les capacités d'actions des acteurs de la lutte contre les exclusions. Une des clés de voûte qui permettraient aux marchés transitionnels de structurer de manière positive les transformations sur le marché du travail, est de redéfinir la nature des contrats de parcours en allant vers plus de droits pour ceux-ci et en les insérant dans le fonctionnement «normal» du marché du travail.

2.3.2. Dynamiques d'acteurs et construction d'une démocratie participative locale

Le PLIE des Graves joue un rôle positif dans l'accompagnement des bénéficiaires des parcours d'insertion et globalement, selon les acteurs de l'économie solidaire, ses instances délibératives bénéficient d'un mode de fonctionnement démocratique.

Un examen des structures et de leur composition montre nettement une segmentation des organes du PLIE. En effet, le Conseil d'administration et le Comité de pilotage sont des fonctions «politiques» dont s'accaparent les élus locaux (le Maire de Bègles et le Président de la CCM) et leurs représentants au sein du territoire du PLIE. La dimension opérationnelle et d'ingénierie est confiée aux directeurs de structures supports de référents (ANPE, Mission Locale, Maison départementale de solidarité et d'insertion, Centre 
communal d'action sociale, Antenne girondine d'insertion). Ils valident les orientations du Conseil d'Administration, lancent les appels d'offres et sélectionnent les candidats à la mise en œuvre. Aux SIAE reviennent les actions de terrain, en contact avec les bénéficiaires au quotidien. Ce mode d'organisation montre que la démocratie locale peut ne pas être participative. Les uns s'accaparent de la vision et les autres l'exécutent selon un cahier des charges validé par les premiers. D'ailleurs, certains des responsables associatifs ne manquent pas de penser que leur cooptation est instrumentale. Ils en concluent que l'émergence d'une démocratie participative locale passe par l'acceptation de contre-pouvoirs aux élus locaux. La question de la légitimité est posée dès lors que des compétences sont reconnues à de telles instances (Blondiaux, 2001). Tout en acceptant l'institutionnalisation de la consultation, de la planification et de la mise en œuvre partagées, il convient de veiller à ce que ces instances aient une durée de vie courte et soient renouvelables régulièrement.

\section{CONCLUSION}

La démocratie participative est une exigence du développement durable qui s'inscrit au cœur des dynamiques des territoires. L'approche de «l'agencéité située » nous a permis, dans ce travail, de comprendre les représentations que les acteurs de l'économie solidaire se font de la démocratie participative à partir de leur partenariat avec le PLIE des Graves, d'évaluer les limites $\mathrm{du}$ fonctionnement démocratique des instances délibératives locales et les performances du modèle en vigueur. Les résultats mettent à nu une segmentation dans la vision de la démocratie participative des acteurs. En effet, le PLIE définit les grandes lignes des politiques publiques locales d'insertion et détient les moyens qu'il redistribue aux SIAE selon une logique à géométrie variable. Cette segmentation est source de frustration de la part de certains acteurs associatifs qui déplorent le mode de fonctionnement selon lequel des acteurs qu'on pourrait qualifier de politiques, en l'occurrence les gestionnaires du PLIE, s'accaparent le leadership et les 
moyens financiers, tout en bénéficiant de résultats qu'ils n'ont pas réalisés. La participation au sens de la théorie de l'agencéité insiste sur la capacité d'organisation intentionnelle, d'action et d'anticipation individuelle et collective. La lutte contre les exclusions, si elle aboutit, traduit un empowerment individuel qui est le résultat d'un empowerment collectif, fruit de la coopération des différents acteurs. Ainsi, collectivement se forge une capacité d'organisation intentionnelle autour du PLIE au travers des acteurs de l'économie solidaire.

Finalement, dans l'échelle d'appréciation de l'ancrage de la démocratie participative de l'école de Chicago, on peut considérer qu'on est dans un système de balancier situé à la fois entre le partage d'informations (phase 3) et la consultation (phase 4) d'une part, et l'association (phase 6) et la délégation de pouvoir (phase 7) d'autre part. Il existe, selon les systèmes de légitimation et de représentation, une différenciation des acteurs dans leurs reconnaissances, les uns bénéficiant de délégation de pouvoir, tandis que les autres se voient confinés à un partage d'informations.

Ceci suggère que la seule volonté politique de mettre en place une dynamique de démocratie participative locale ne suffit pas à la faire vivre. La démocratie participative locale est un apprentissage, un processus qui s'inscrit dans la durée et doit être le fruit d'une maturation et d'une prise de conscience locale. C'est pourquoi les critiques exprimées par les différents acteurs rencontrés montrent que la démocratie participative locale doit être conçue comme un idéal-type. Mais, nous pensons que ces réserves n'invalident pas les performances de l'instance PLIE dans un processus de démocratisation. A contrario, de meilleurs résultats ne dépendraient-ils pas du respect de l'autonomie et de la liberté de telles instances délibératives?

\section{BIBLIOGRAPHIE}

ARNSTEIN S., (1969), «A ladder of citizen participation », Journal of the American Institute of Planners, $\mathrm{N}^{\circ} 35$, vol. 4, pages $216-224$. 
AYADALOT P., (1986). Milieux innovateurs en Europe, Gremi.

BENKO G., LIPIETZ A., (1992), Les régions qui gagnent, PUF.

BLONDIAUX L., (2001), «Démocratie locale et participation citoyenne : la promesse et le piège », Mouvements, $\mathrm{N}^{\circ} 18$, pp. 44-51.

BRAILLON C., (2007), "Démocratie participative locale. Introduction », Revue Territoires, Les pratiques $\mathrm{N}^{\circ} 6$, CEDIS, ADELS, page 5.

CEDIS, ADELS (dir.), (2007), La démocratie participative locale, Revue Territoires, Les pratiques $\mathrm{N}^{\circ} 6$, ADELS.

CHOCHOY N., (à paraître), «Prise de parole et économie solidaire. Les apports d'Albert $O$. HIRSCHMAN à la notion de pratiques d'économie solidaire », Colloque international «Économie sociale et solidaire: nouvelles approches et dynamiques territoriales », France, 29-30 septembre 2008, 11 pages. CLING J-P., (2003), Une analyse critique du discours de la Banque mondiale sur la lutte contre la pauvreté in Cling J-P., M. Razafindrakoto et Roubaud F. (dir.), 2003, Les nouvelles stratégies internationales de réduction de la pauvreté, Economica, pages 25-56.

CROZIER M., FRIEDBERG E., (1977), L'Acteur et le système, Seuil, coll. Points essais.

DAGHRI T., ZAOUAL H., (2008), Économie solidaire et développement local. Vers une démocratie située, coll. Horizon pluriel, GREL, L'Harmattan.

DERCON S., (2004), « Analyse microéconomique de la pauvreté et des inégalités : l'arbitrage équité-efficacité revisité », Afrique contemporaine, Automne 2004, pages 73-97.

DI MEO G., (1998), Géographie sociale et territoires, Nathan.

DUBOIS J-L., BROUILLET A-S., BAKHSHI P., DURAY-SOUNDRON C (dir.), (2008), Repenser l'action collective. Une approche par les capabilités, coll. Ethique économique, L'Harmattan \& Réseau IMPACT.

EDGERTHON J. ET ALII, (2000), Participatory processes in The Poverty Reduction Strategy, Poverty Reduction Strategy Sourcebook, World Bank. 
HIRSCHMAN A. O., (1995), Défection et prise de parole, Fayard.

MACHRAFI M., (2008), Introduction au paradigme des sites. Epistémologie et concepts, Revue Repères et Perspectives $\mathrm{n}^{\circ} 11$, pages 81-92.

NASSBAUM M., (2000), Women and Human Development: The Capabilities Approach, Cambridge University Press.

NDIAYE A., (à paraître), «Économie solidaire, insertion et marchés transitionnels du travail territorialisés. Quelques conclusions tirées de l'étude de cas du PLIE des Graves », Colloque international «Économie sociale et solidaire: nouvelles approches et dynamiques territoriales », France, 29-30 septembre 2008, 16 pages. NDIAYE A., (2009), «L'initiative PPTE est-elle efficace pour la réduction de la pauvreté ? Étude de cas du Sénégal », in Daffé G., Diagne A. (dir.) Le Sénégal face aux défis de la pauvreté. Les oubliés de la croissance, CRES-Karthala-CREPOS, collection Hommes et Sociétés, pages 301-322.

NDIAYE A., (2008), «Institutions des territoires et mondialisation. L'étude de cas du PLIE des Graves », in Christen Jakob M., Reichmuth J. (Ed.), Community development: local and global challenges, pages 201208, École supérieure du travail social, Lucerne.

PECQUEUR B., (2000), Le développement local. Pour une économie des territoires, Alternatives ÉconomiquesSYROS.

RAIBAUD Y., (à paraître), « Le Centre rural d'animation Culturelle de l'Entre-deux-Mers (1982-2001). Un exemple de développement local mené par une association d'économie sociale et solidaire » Colloque international «Économie sociale et solidaire: nouvelles approches et dynamiques territoriales », France, 29-30 septembre 2008

ROSANVALLON P., (1995), La nouvelle question sociale. Repenser l'État-providence, Seuil.

SEN A., (2000), Repenser l'inégalité, Seuil.

ZAOUAL H., (1999), « Théorie des sites et organisation «économique»", in Latouche et al., Critique de la raison économique. Introduction à la théorie des sites symboliques, L'Harmattan, pages 79-109. 